\title{
AÇÕES GERENCIAIS DE ENFERMAGEM NOS SERVIÇOS DE REFERÊNCIA EM CARDIOLOGIA
}

\author{
MANAGED NURSING ACTIONS IN CARDIOLOGY REFERENCE SERVICES \\ Thalisson Dheison Alves Cássia ${ }^{1} *$ Dênison Pereira da Silva ${ }^{2} *$ Daniel Batista Conceição dos \\ Santos $^{3} *$ Michelle Santana Prata ${ }^{4} *$ Luana da Conceição Costa Cardoso ${ }^{5} *$ Edielson dos \\ Anjos Costa ${ }^{6}$
}

\section{RESUMO}

Os serviços de cardiologia são setores críticos devido à imprevisibilidade de ocorrências e ritmos altamente acelerados. Logo, o gerenciamento de enfermagem é algo imprescindível e que influencia diretamente na qualidade da assistência. Trata-se de uma revisão integrativa da literatura, que teve por objetivo conhecer publicações de enfermagem e os impactos das ações gerenciais desses para a qualificação dos serviços de cardiologia. Foram utilizados 10 artigos das bases de dados LILACS, PubMed e SciELO. Utilizaram-se os descritores "Gestão em Saúde", "Enfermagem", "Serviços Hospitalares" e "Cardiologia" de forma cruzada. A seleção dos artigos foi realizada nos meses de outubro e novembro de 2018. Os critérios de inclusão foram: artigos produzidos por enfermeiros; publicados no período de 2009-2018; nos idiomas português, espanhol e inglês; e disponíveis eletronicamente na íntegra. Os dados foram organizados e tabulados para análise. Os resultados constataram que a maioria dos artigos que compuseram essa revisão foi de natureza qualitativa e publicados em revistas nacionais de enfermagem. Destacaram-se como ações gerenciais as práticas de tomada de decisão, liderança, comunicação, treinamentos, capacitações, alocação de recursos humanos e materiais. O trabalho do enfermeiro, em unidades cardiológicas públicas, representa um desafio, o que reforça a necessidade de estudos que comprovem sua relevância para promoção de melhorias contínuas da assistência.

Palavras-chave: Gestão em Saúde; Enfermagem; Serviços Hospitalares; Cardiologia.

\begin{abstract}
Cardiology services are critical sectors due to the unpredictability of occurrences and highly accelerated rhythms. Therefore, nursing management is essential and has a direct influence on the quality of care. This is an integrative literature review, which aimed to learn about nursing publications and the impacts of their managerial actions for the qualification of cardiology services. 10 articles from the LILACS, PubMed and SciELO databases were used. The descriptors "Health Management", "Nursing", "Hospital Services" and "Cardiology" were used crosswise. The selection of articles was carried out in the months of October and November 2018. The inclusion criteria were: articles produced by nurses; published in the period 2009-2018; in Portuguese, Spanish and English; and available electronically in full. The data were organized and tabulated for analysis. The results found that most of the articles that comprised this review were of a qualitative nature and published in national nursing journals. Managerial practices stood out as practices of decision making, leadership, communication, training, qualification, allocation of human and material resources. The work of nurses in public cardiology units represents a challenge, which reinforces the need for studies that prove their relevance for promoting continuous improvements in care.
\end{abstract}

Keywords: Management in Health; Nursing; Hospital Services; Cardiology.

\footnotetext{
${ }^{1}$ Enfermeiro. Especialista em Saúde Mental pela Universidade Federal de Sergipe - UFS. Especialista em Enfermagem Cardiológica pela Universidade Tiradentes - UNIT e Graduando em Medicina pela Universidade Tiradentes - UNIT. http://orcid.org/0000-00031640-9399. Email:

${ }^{2}$ Enfermeiro. Doutor em Saúde e Ambiente pela Universidade Tiradentes - UNIT. http://orcid.org/0000-0002-8518-1757

${ }^{3}$ Enfermeiro. Doutorando em Cardiologia pela Universidade de São Paulo - USP. http://orcid.org/0000-0002-8204-4714

${ }^{4}$ Enfermeira. Especialista em Enfermagem Cardiológica pela Universidade Tiradentes - UNIT. http://orcid.org/0000-0001-80500707

${ }^{5}$ Enfermeira. Pós Graduanda em Segurança do Paciente pela Faculdade de Venda Nova Imigrante - FAVENI. http://orcid.org/00000003-1125-8552

${ }^{6}$ Médico. Graduado em Medicina pela Universidade Federal de Sergipe - UFS. http://orcid.org/0000-0001-8873-2908.
} 


\section{INTRODUÇÃO}

As Doenças Cardiovasculares (DCV) estão entre os principais problemas de saúde pública mundial, em razão da sua elevada incidência que provoca 17,9 milhões de mortes todos os anos, das quais 7,4 milhões (13\%) são causadas por doenças coronárias e 6,7 milhões (12\%), acidentes vasculares cerebrais $^{(1-2)}$.

De acordo com a American Heart Association, em 2017 as DCV foram a causa subjacente de quase 801.000 óbitos nos Estados Unidos ${ }^{(3)}$. Do mesmo modo no Brasil, foram estimados 383.961 óbitos, $28 \%$ do total, sendo a segunda causa de morte em adultos jovens e a primeira em pessoas acima de 60 anos, o que geraram custos de mais 37 bilhões ao sistema de saúde em $2015^{(4-5-6)}$.

Essa configuração epidemiológica, associada à evolução tecnológica, das políticas públicas e dos serviços de saúde vem instigando pesquisadores da enfermagem, sobretudo no que concerne à dinâmica de trabalho, mobilização de recursos, gestão de processos para o alcance das metas e consequentemente propostas para qualificação dos serviços ${ }^{(7-8)}$.

Nesse contexto, dentre os setores da saúde, as unidades cardiovasculares, representam um dos serviços mais críticos em relação à qualidade do atendimento, devendo ser providas de recursos humanos e materiais necessários para implantação de uma assistência efetiva, haja vista a especificidade dos pacientes atendidos, exigindo da equipe multidisciplinar aprimoramento científico, desenvolvimento de habilidades e dinamização do cuidado ${ }^{(9)}$.

$\mathrm{O}$ enfermeiro, enquanto gerente ou líder de equipe, necessita de conhecimentos teóricos e práticos que possam fundamentar e habilitar o desenvolvimento das atividades de maior complexidade, as quais envolvem a capacitação, atualização, revitalização ou o reconhecimento das aptidões e do desenvolvimento de potenciais dos trabalhadores de enfermagem como prática que emerge do trabalho para favorecer melhores ações de cuidado no contexto institucional $^{(10-11)}$.

No entanto, o gerenciamento é visualizado pelos enfermeiros, muitas vezes, como uma atividade burocrática e desvinculada da produção do cuidado, o que é caracterizado pela fragmentação do trabalho com separação entre concepção e execução, controle gerencial do processo de produção associado à rígida hierarquia, a racionalização da estrutura administrativa, a impessoalidade nas relações interpessoais e a ênfase em sistemas de procedimentos e rotinas ${ }^{(12-13)}$.

Constata-se, portanto, a relevância desta pesquisa, voltada para destacar as competências do enfermeiro de desenvolver ações gerenciais, no contexto dos serviços de referência cardiovascular, com ênfase na qualificação da assistência e justifica-se pelo interesse de compreender a aplicabilidade 
dessas ações no âmbito dos serviços públicos de saúde, os quais atendem boa parcela da população no Brasil.

Assim, esta pesquisa teve como objetivos conhecer as ações gerenciais do enfermeiro nos serviços de referência em cardiologia e destacar o trabalho do enfermeiro enquanto gerente de unidade cardiovascular.

\section{MÉTODO}

Utilizou-se como recurso metodológico a revisão integrativa que se constitui como ferramenta importante para análise de evidências na literatura de forma ampla e sistemática para divulgar os resultados dos estudos, contribuindo para apontar problemáticas que podem ser solucionadas com evidências descobertas em outras pesquisas $^{(14)}$.

A construção desta revisão estruturouse com base nas seguintes etapas: identificação do tema, formulação da questão condutora; estabelecimento dos critérios de elegibilidade; levantamento dos estudos nas bases de dados; avaliação e análise crítica dos estudos selecionados; categorização, avaliação e interpretação dos resultados e apresentação da síntese na revisão integrativa ${ }^{(15)}$.

Nesta perspectiva, elaborou-se a questão que norteou este trabalho: Quais são as ações gerenciais do enfermeiro para qualificação dos serviços de cardiologia, descritos na literatura?

Adotou-se como critérios de inclusão artigos originais em idioma português, inglês e espanhol, disponíveis na íntegra, nas bases de dados Literatura Latino-Americana e do Caribe em Ciências da Saúde (LILACS), National Library of Medicine (PubMed) e Scientific Electronic Library Online (SciELO), no período de 2009 a 2018 que abordassem as principais ações gerenciais realizadas pelos enfermeiros no contexto das unidades cardiovasculares. Foram excluídos os artigos de revisão bibliográfica, duplicados nas bases de dados ou não compreendidos no período estabelecido. A pesquisa ocorreu nos meses de outubro e novembro de 2018 e foi utilizado o software Mendeley para gerenciamento das referências.

Para operacionalização desta pesquisa utilizou-se a recomendação PRISMAPrincipais Itens para Relatar Revisões Sistemáticas e Meta-análises ${ }^{(16)}$, conforme fluxograma da figura 1. 
Figura 1 - Fluxograma da estratégia de busca nas bases de dados LILACS, PubMed e SciELO, Aracaju/SE, 2018

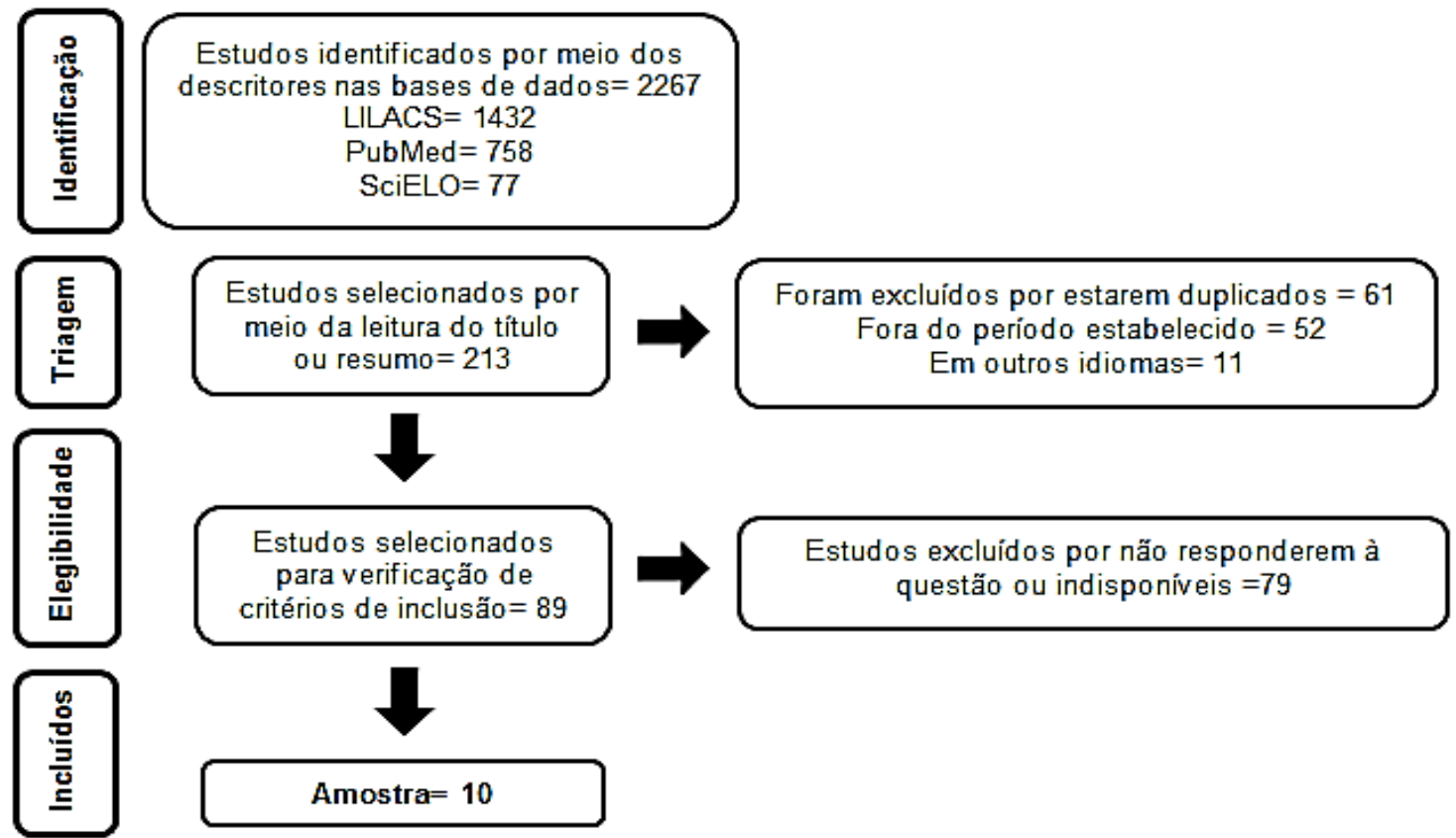

Fonte: Dados da pesquisa.

A busca iniciou-se com a seleção dos descritores utilizados nas bases de dados, por meio da terminologia DeCS/MeSH: gestão em saúde/ management in health, enfermagem/ nursing, serviços hospitalares/ hospital services e cardiologia/ cardiology.

A estratégia de busca foi ("gestão em saúde" AND "enfermagem" AND “cardiologia" OR "gestão em saúde" AND "serviços hospitalares" AND "enfermagem") e ("Health management" AND "nursing" AND "cardiology" OR "health management" AND "hospital services" AND "nursing").

A seleção dos artigos foi executada de modo pareado entre os autores mediante leitura dos títulos e resumo dos estudos que obedecessem aos critérios de inclusão. Em seguida os estudos foram avaliados em conjunto, de forma exploratória, com base em um formulário estruturado e validado por Ursi (2005), o qual discriminava título, autores, ano, país, objetivos, método e principais resultados. Ao final foram selecionados 4 artigos da LILACS; 3 PubMed e 3 da SciELO. Dessa forma, a amostra foi composta de 10 artigos.

A diversidade de abordagens dos estudos, cenários, ações realizadas e os resultados impediram a realização de uma revisão sistemática formal ou meta-análise, portanto, esta revisão apresentou os resultados de forma descritiva e narrativa.

É importante ressaltar que esta pesquisa, por possuir caráter bibliográfico, não foi submetida ao Comitê de Ética em Pesquisa da Universidade Tiradentes 
(CEP/Unit), todavia, garantiu a autoria das ideias utilizadas na sua construção conforme normativa da NBR 10520 (exigências acerca das citações) e NBR 6023 (estabelece os parâmetros de referências), além da Lei 12.853/2013 (gestão coletiva dos direitos autorais).

\section{RESULTADOS}

A tabela 1 apresenta a distribuição dos estudos conforme autor, ano de publicação, país de origem, periódico, método, participantes e principais ações do enfermeiro gerente.

Tabela 1 - Síntese dos artigos que compuseram a revisão integrativa. Aracaju/SE, 2018

\begin{tabular}{|c|c|c|c|c|}
\hline Autor/ano/país & Periódico & Método & Participantes & $\begin{array}{c}\text { Ações gerenciais de } \\
\text { enfermagem }\end{array}$ \\
\hline $\begin{array}{l}\text { Vieira LC, Contrin } \\
\text { LM, Rol JD, Conte } \\
\text { HD, Lima AR, } \\
\text { Castro ED, Santos } \\
\text { ML, 2009(17) } \\
\text { Brasil }\end{array}$ & $\begin{array}{l}\text { Arquivos de } \\
\text { Ciências da } \\
\text { Saúde }\end{array}$ & $\begin{array}{l}\text { Exploratório } \\
\text { Descritivo }\end{array}$ & $\begin{array}{c}56 \\
\text { Enfermeiros }\end{array}$ & $\begin{array}{l}\text { Previsão e provisão de materiais, } \\
\text { confecção de escalas, reuniões } \\
\text { com chefias, educação } \\
\text { permanente, supervisão da } \\
\text { equipe. }\end{array}$ \\
\hline $\begin{array}{c}\text { Kobayashi RM, } \\
\text { Vendramini AB, } \\
\text { Ayoub AC, } \\
2010^{(7)} \\
\text { Brasil }\end{array}$ & $\begin{array}{l}\text { Revista da } \\
\text { Rede de } \\
\text { Enfermagem } \\
\text { do Nordeste }\end{array}$ & $\begin{array}{c}\text { Descritivo } \\
\text { Exploratório } \\
\text { Documental }\end{array}$ & $\begin{array}{c}7 \\
\text { Enfermeiras }\end{array}$ & $\begin{array}{l}\text { Planeja o espaço físico, } \\
\text { dimensiona recursos humanos e } \\
\text { materiais. }\end{array}$ \\
\hline $\begin{array}{c}\text { Linch GF, Guido } \\
\text { LD, Fantin SD, } \\
2010^{(18)}\end{array}$ & $\begin{array}{c}\text { Texto } \\
\text { Contexto de } \\
\text { Enfermagem } \\
\end{array}$ & Transversal & $\begin{array}{c}63 \\
\text { Enfermeiros }\end{array}$ & $\begin{array}{l}\text { Treinamento e supervisão da } \\
\text { equipe de enfermagem. }\end{array}$ \\
\hline $\begin{array}{c}\text { Oliveira EB, Souza } \\
\text { NV, 2012 }{ }^{(19)} \\
\text { Brasil }\end{array}$ & $\begin{array}{l}\text { Revista de } \\
\text { Enfermagem } \\
\text { da UERJ }\end{array}$ & $\begin{array}{l}\text { Descritivo } \\
\text { Qualitativo }\end{array}$ & $\begin{array}{c}7 \\
\text { Enfermeiros }\end{array}$ & $\begin{array}{l}\text { Treinamento } \text { em serviço, } \\
\text { elaboração de rotinas e } \\
\text { protocolos, realização de } \\
\text { procedimentos de maior } \\
\text { complexidade e supervisão da } \\
\text { equipe. }\end{array}$ \\
\hline $\begin{array}{l}\text { Hickey PA, 2012(20) } \\
\text { Estados Unidos }\end{array}$ & $\begin{array}{c}\text { American } \\
\text { Journal of } \\
\text { Critical Care }\end{array}$ & Transversal & $\begin{array}{c}150 \\
\text { Enfermeiros }\end{array}$ & $\begin{array}{l}\text { Padronização da comunicação, } \\
\text { liderança, criação de listas de } \\
\text { verificação, viabilização do } \\
\text { processo do cuidado. }\end{array}$ \\
\hline $\begin{array}{c}\text { Driscoll A, Currey J, } \\
\text { Allen JA, George M, } \\
\text { Davidson PM, 2014 } \\
\text { (21) } \\
\text { Austrália } \\
\end{array}$ & $\begin{array}{c}\text { Australian } \\
\text { Critical Care }\end{array}$ & Transversal & $\begin{array}{c}24 \\
\text { Enfermeiros }\end{array}$ & $\begin{array}{l}\text { Realização de procedimentos de } \\
\text { maior complexidade. }\end{array}$ \\
\hline $\begin{array}{l}\text { Aruto GC, Lanzoni } \\
\text { GMM, Meirelles } \\
\text { BH, 2016 } \\
\text { Brasil }\end{array}$ & $\begin{array}{c}\text { Cogitare } \\
\text { Enfermagem }\end{array}$ & $\begin{array}{l}\text { Descritivo } \\
\text { Qualitativo }\end{array}$ & $\begin{array}{c}14 \\
\text { Enfermeiros }\end{array}$ & $\begin{array}{l}\text { Supervisão da equipe, educação } \\
\text { permanente, comunicação, } \\
\text { liderança, gestão da unidade e } \\
\text { capacitação. }\end{array}$ \\
\hline $\begin{array}{c}\text { Koerich C, Erdmann } \\
\text { AL, } 2016^{(23)} \\
\text { Brasil }\end{array}$ & $\begin{array}{c}\text { Revista } \\
\text { Brasileira de } \\
\text { Enfermagem }\end{array}$ & Qualitativo & $\begin{array}{c}22 \\
\text { Profissionais }\end{array}$ & $\begin{array}{l}\text { Discutir ações de cuidar com a } \\
\text { equipe, gerenciar as práticas de } \\
\text { educação permanente } \\
\text { supervisão do cuidado de } \\
\text { enfermagem, identificação das } \\
\text { necessidades }\end{array}$ \\
\hline
\end{tabular}




\begin{tabular}{|c|c|c|c|c|}
\hline & & & & $\begin{array}{l}\text { aperfeiçoamento profissional e } \\
\text { orientação na equipe. }\end{array}$ \\
\hline $\begin{array}{c}\text { Silva VL, Camelo } \\
\text { SH, Soares MI, } \\
\text { Resck ZM, Chaves } \\
\text { LD, Santos FC, Leal } \\
\text { LA, 2017 } \\
\text { Brasil }\end{array}$ & $\begin{array}{l}\text { Revista da } \\
\text { Escola de } \\
\text { Enfermagem } \\
\text { da USP }\end{array}$ & $\begin{array}{l}\text { Transversal } \\
\text { Descritivo } \\
\text { Correlacional }\end{array}$ & $\begin{array}{c}84 \\
\text { Enfermeiros }\end{array}$ & $\begin{array}{l}\text { Promovem práticas de liderança } \\
\text { para capacitar, encorajar, traçar } \\
\text { o caminho, desafiar o processo e } \\
\text { inspirar uma visão } \\
\text { compartilhada, proporciona o } \\
\text { trabalho em equipe, ajuda a criar } \\
\text { um clima de confiança, partilha } \\
\text { poder e valoriza seus liderados. }\end{array}$ \\
\hline $\begin{array}{c}\text { La-Sala R, } \\
\text { Dicembrino RB, } \\
\text { Dall'Argine S, } \\
\text { Baiguera M, } \\
\text { Gazzotti L, Gubin E, } \\
\text { Rondini A, Mamei I, } \\
\text { 2017 }{ }^{(25)} \\
\text { Itália }\end{array}$ & $\begin{array}{l}\text { Acta Biomed } \\
\text { for Health } \\
\text { Professions }\end{array}$ & $\begin{array}{l}\text { Observacional } \\
\text { multicêntrico } \\
\text { Quantitativo }\end{array}$ & $\begin{array}{c}154 \\
\text { Enfermeiros }\end{array}$ & $\begin{array}{l}\text { Educação permanente, } \\
\text { supervisão, tomada de decisão e } \\
\text { treinamentos. }\end{array}$ \\
\hline
\end{tabular}

Fonte: Dados da pesquisa.

No mesmo sentido, quanto o ano de

No que se refere a origem dos estudos, 7 artigos foram publicados no Brasil, em periódicos de enfermagem ou interdisciplinar ${ }^{(7,17-19,22-24,)}$. Estados Unidos, Austrália e Itália contribuíram com um estudo $\operatorname{cada}^{(20-21,25)}$. publicação, verificou-se que os anos de 2010, 2012, 2016 e 2017 destacaram-se com maior número de artigos, 2 ao ano. 2009 e 2014 contribuíram com uma publicação. Estudos referentes aos anos de 2011, 2013 e 2015 (Gráfico1).

Gráfico 1 - Distribuição dos artigos, segundo ano de publicação, Aracaju/SE, 2018

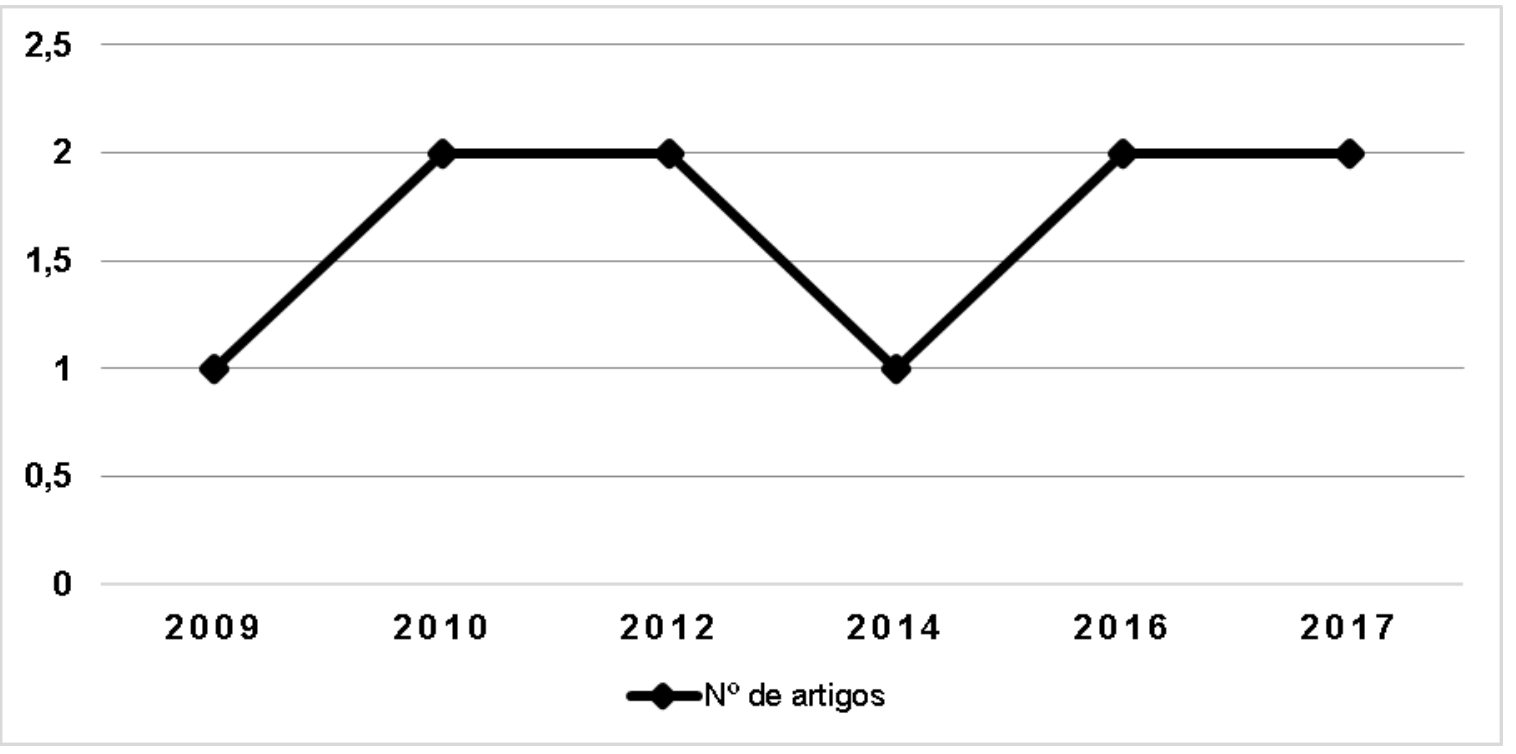

Fonte: Dados da pesquisa. 
Os artigos selecionados foram socializados em 10 periódicos distintos, com destaque para as revistas Arquivos de Ciências da Saúde, Revista da Rede de Enfermagem do Nordeste, Texto Contexto de Enfermagem, Revista de Enfermagem da UERJ, American Journal of Critical Care, Australian Critical Care, Cogitare Enfermagem, Revista Brasileira de Enfermagem, Revista da Escola de Enfermagem da USPe Acta Biomed for Health Professions.

Foram identificados estudos onde houve a participação de 7 enfermeiros ${ }^{(7,19)}$, já La-Sala et al. $(2017)^{(25)}$ tiveram em sua pesquisa a participação de 154 enfermeiros. A análise da metodologia de pesquisa dos artigos, permitiu identificar que 9 artigos utilizaram a abordagem metodológica qualitativa e apenas um a quantitativa. Nos estudos de abordagem qualitativa, os métodos foram: descritivo, documental, transversal.

A presente investigação evidenciou que os enfermeiros gerentes possuíam idade entre 23 a 62, predominância do sexo feminino, tempo de formação acima de 5 anos, pós-graduação, não necessariamente na área de cardiologia, havendo na área de urgência e emergência, UTI e gestão ${ }^{(17-}$ $18,20,24)$.

Observou-se também a carência de enfermeiros gerentes especializados em cardiologia, o que demonstra a necessidade de qualificação desses profissionais para o atendimento em alta complexidade cardiovascular, em razão das demandas que o setor exige nas ações de capacitação e qualificação da equipe de enfermagem, organização e desenvolvimento dos processos de trabalho do setor, atividades de suporte assistencial, a exemplo do gerenciamento de risco, segurança do paciente e gestão para qualidade total $^{(20-21)}$.

\section{DISCUSSÃO}

A gestão dos serviços de cardiologia tem como foco principal a organização da assistência integral, isto é, o planejamento de ações compartilhadas de modo que a equipe de enfermagem, sob a liderança do enfermeiro, desenvolva o processo de trabalho com eficiência e qualidade, a fim de satisfazer as necessidades de segurança e bem-estar do paciente assistido ${ }^{(22)}$.

Assim, para os autores, as ações gerenciais essenciais ao trabalho do enfermeiro gerente de unidade cardiovascular estão relacionadas a tomada de decisão, comunicação, liderança, provisão de recursos materiais, dimensionando de recursos humanos, supervisão, elaboração de protocolos, educação permanente, entre outras ${ }^{(7,17-18,20)}$.

Quanto ao gerenciamento do serviço, um estudo realizado com 7 enfermeiras que atuam em uma instituição governamental estadual de referência na área cardiovascular refere que o planejamento e a tomada de decisão, como funções atribuídas ao enfermeiro, auxiliam no desenvolvimento da gestão do $\operatorname{serviço}^{(7)}$, em virtude disso, promove o uso apropriado dos equipamentos, garantindo a eficácia e custo no processo de seleção e treinamento da força de trabalho e na realização de procedimentos e práticas de alta complexidade ${ }^{(18-19)}$.

A comunicação influencia diretamente na efetivação de protocolos institucionais, supervisão e no processo de liderança, uma vez que propicia ao enfermeiro transferir e receber informações e 
conhecimentos, organizar seu serviço e delinear objetivos junto à sua equipe. Desta maneira, o êxito do líder está relacionado com a habilidade de comunicar, sendo esta essencial para reduzir os eventos adversos e garantir um cuidado seguro, além de facilitar o alcance de metas previamente estabelecidas $^{(20,22)}$.

Desse modo, pode-se constatar que a liderança é uma ação importante no trabalho do enfermeiro gerente dos serviços de cardiologia, em razão de se constituir ferramenta de promoção de melhorias do cuidado, que auxilia na avaliação, planejamento e comunicação dos processos de trabalho do setor.

Em relação ao dimensionamento dos recursos humanos, Kobayashi, Silva e Ayoub $(2010)^{(7)}$ apontam que essa ação viabiliza o provimento de pessoal necessário para suprir as demandas de assistência ao cliente. Possibilita ainda, avaliar a carga de trabalho presente na unidade e assim, planejar o quantitativo ideal de trabalhadores da enfermagem para prover $\mathrm{o}$ cuidado, tornando essa atividade como requisito essencial para mensurar a qualidade da assistência prestada.

Do mesmo modo, o gerenciamento de recursos materiais na unidade cardiológica constitui prática eficaz no controle de qualidade e desperdício, devido ao alto custo destes materiais e a dificuldade de reposição ${ }^{(22-23)}$, principalmente nas instituições públicas onde os recursos são escassos. Logo, o uso das tecnologias e inovações dos equipamentos são imprescindíveis para assistência, pois os insumos e equipamentos obsoletos aumentam a possibilidade de erros, riscos à saúde do trabalhador e infortúnios a segurança do paciente ${ }^{(18-19)}$.
Como consequência desses fatos, verificase a necessidade de o enfermeiro gerente participar da compra e distribuição de materiais, intermediando os contatos entre os níveis institucionais e os setores responsáveis, sendo esse aspecto um dos quesitos fundamentais do trabalho do enfermeiro que garante as melhorias contínuas da assistência e a gestão para qualidade total.

No contexto dos serviços de cardiologia, foi unânime nos estudos a concepção de que a ausência de capacitação prejudica a assistência. Em detrimento dessa circunstância, é imprescindível que o enfermeiro gerente assuma o desafio de planejar treinamentos de educação continuada, mediante implantação de programas específicos que abordem conhecimentos práticos acerca do manuseio dos equipamentos utilizados nos procedimentos, as condutas requeridas nas situações do cotidiano, bem como no alcance das metas instituídas ${ }^{(18,23,25)}$.

Nesse sentido, a implantação de educação permanente nos serviços de cardiologia, sob a perspectiva da gerência de enfermagem, oportuniza os profissionais a vivenciar a aprendizagem, conforme a realidade do setor e o cotidiano das atividades, corroborando para qualificação desses serviços.

\section{CONCLUSÃO}

A partir de uma revisão integrativa, buscou-se conhecer as ações gerenciais de enfermagem nos serviços de cardiologia. Logo, o estudo mostrou-se eficaz para fornecer subsídios na construção do perfil do enfermeiro gerente, ou seja, são profissionais capazes de impulsionar e 
mobilizar as práticas de cuidado no contexto desses serviços.

Assim, diante da complexidade das ações gerenciais, o enfermeiro deve estar preparado para conduzir a equipe e os cuidados aos pacientes, sendo essencial competências e habilidades nas práticas de tomada de decisão, liderança, comunicação, treinamentos e capacitações, por meios da educação permanente e alocação de recursos humanos e materiais.

Dessa forma, o desenvolvimento do trabalho do enfermeiro, principalmente em unidades cardiológicas públicas, representa um grande desafio, afinal, não dispõem de recursos humanos e materiais suficientes para implantação das estratégias capazes de atenuar ou eliminar os problemas identificados nas unidades. O que reforça a necessidade de mais estudos que comprovem sua relevância para promoção de melhorias contínuas da assistência.

\section{REFERÊNCIAS}

1. Organização Mundial de Saúde - OMS [Internet]. Doenças Cardiovasculares. 2017 [acesso em 08 nov 2018]. Disponível em: https://www.paho.org/bra/index.php?option= com_ content\&view $=$ article $\&$ id $=5253:$ doencascardiovasculares $\&$ Itemid $=839$

2. Silveira IH, Junger WL. Espaços verdes e mortalidade por doenças cardiovasculares no município do Rio de Janeiro. Rev Saude Publica. 2018; 52(1):49

3. Benjamin EJ, Blaha MJ, Chiuve SE. On behalf of the American Heart Association Statistics Committee and Stroke Statistics Subcommittee. Heart disease and stroke statistic - 2017 update: a report from the American Heart Association. Circulation. 2017; 135(10):e146-e603.
4. Lamendola C, Champagne MA, Worel JN, Coke LA. Access to Innovative Medicine: A Preventive Cardiovascular Nurses Association Position Statement to Reduce the Barriers. J Cardiovasc Nurs. 2018; 33(3):199201.

5. Brasil. Ministério da Saúde [Internet]. Doenças cardiovasculares são a principal causa de morte no mundo. 2017 [acesso em 02 fev 2020]. Disponível em: http://www.brasil.gov.br/noticias/saude/ 2017/09/doencas-cardiovasculares-saoprincipal-causa-de-morte-no-mundo

6. Siqueira AD, Siqueira-Filho AG, Land MG. Análise do impacto econômico das doenças cardiovasculares nos últimos cinco anos no Brasil. Arq Bras Cardiol. 2017; 109(1):39-46.

7. Kobayashi RM, Vendramini AB, Ayoub AC. Gerenciando dificuldades para acreditação hospitalar em hospital cardiovascular. Rev Rene. 2010; 11(4):19-28.

8. Koerich C, Erdmann AL. Significados atribuídos pela equipe de enfermagem sobre educação permanente em uma instituição cardiovascular. Rev Rene. 2016; 17(1):93102.

9. Aguiar DF, Conceição-Stipp MA, Leite JL, Mattos VZ, Andrade KB. Gerenciamento de enfermagem: situações que facilitam ou dificultam o cuidado na unidade coronariana. Aquichan. 2010; 10(2):115-31.

10. Giordani JN, Bisogno SB, Silva LA. Percepção dos enfermeiros frente às atividades gerenciais na assistência ao usuário. Acta Paul Enferm. 2012; 25(4):5116.

11. Chaves LD, Tanaka OY. O enfermeiro e a avaliação na gestão de Sistemas de Saúde. Rev Esc Enferm USP. 2012; 46(5):1274-8.

12. Santos JLG, Silva RM, Prochnow AG, Beck CLC, Leite JL. O exercício de liderança por uma enfermeira em um contexto de organização do trabalho em saúde e 
enfermagem: algumas considerações. Rev Enferm UFPE On Line. 2009; 3(4):433-39.

13. Camponogara S, Backes VM. Gerenciar em enfermagem: uma reflexão à luz das idéias de Humberto Maturana. Revista Mineira de Enfermagem. 2007; 11(3):303-10.

14. Ramalho Neto JM, Marques DK, Fernandes MD, Nóbrega MM. Meleis' Nursing Theories Evaluation: integrative review. Rev Bras Enferm. 2016; 69(1):162-8.

15. Botelho LL, Cunha CC, Macedo M. The integrative review method in organizational studies. Rev Eletr Gestão Soc. 2011; 5(11):121-36.

16. Galvão TF, Pansani TD, Harrad D. Principais itens para relatar Revisões sistemáticas $\mathrm{e}$ Meta-análises: A recomendação PRISMA. Epidemiol Serv Saúde. 2015; 24(2):335-42.

17. Vieira LC, Contrin LM, Rol JD, Conte HD, Lima AR, Castro ED, Santos ML. Dificuldades e necessidades da equipe de enfermagem em serviços de hemodinâmica e angiografia. Arq Ciênc Saúde. 2009; 16(1):21-5.

18. Linch GF, Guido LD, Fantin SD. Enfermeiros de unidades de hemodinâmica do Rio Grande do Sul: perfil e satisfação profissional. Text \& Context Enfermagem. 2010; 19(3):488-95.

19. Oliveira EB, Souza NV. Estresse e inovação tecnológica em unidade de terapia intensiva de cardiologia: tecnologia dura. Rev Enferm UERJ. 2012; 20(4):457-62.

20. Hickey PA. Intensive Cardiovascular Care in the United States: Nursing and Organizational Characteristics. Am J Crit Care. 2012; 21(4):242-50.

21. Driscoll A, Currey J, Allen JA, George M, Davidson PM. New cardiac models of care reduce patient access to specialist nurses: A Victorian cross-sectional pilot study. Aust Crit Care. 2014; 27(1):17-27.

22. Aruto GC, Lanzoni GMM, Meirelles BH. Melhores práticas no cuidado à pessoa com doença cardiovascular: interface entre liderança e segurança do paciente. Cogitare Enferm. 2016; 21(5)1-9.

23. Koerich C, Erdmann AL. Gerenciando práticas educativas para o cuidado de enfermagem qualificado em cardiologia. Rev Bras Enferm. 2016; 69(5):872-80.

24. Silva VL, Camelo SH, Soares MI, Resck ZM, Chaves LD, Santos FC, Leal LA. Práticas de liderança em enfermagem hospitalar: uma self de enfermeiros gestores. Rev Esc Enferm USP. 2017; 51(1):1-8.

25. La-Sala R, Dicembrino RB, Dall'Argine S, Baiguera M, Gazzotti L, Gubin E, Rondini A, Mamei I. Nurse training in self-management of patients with cardiovascular diseases: a multicentre observational study. Acta Biomed. 2017;88(Suppl 5):22.

Submissão: 2021-01-05

Aprovado: 2021-03-15 